\title{
Morphometric Comparison of Human Nerve Cells: Special Sensory System
}

\author{
By
Eiji TAKAOKI, Naoko NONAKA, Noboru GOTO, Masataka SUZUKI, Jun GOTO and Masakazu SHIBATA

Department of Anatomy, Showa University School of Medicine, Tokyo 142-8555, Japan

- Received for Publication, January 5, 2005 -

Key Words: Cell size, Image-analyzer, Morphology, Morphometry, Special sense

\begin{abstract}
Summary: We compared morphometric data on various nerve cells in the human special sensory system using the modified Klüver-Barrera staining method with an extremely minimized shrinkage ratio and an image-analyzer. According to the measurement data of cell-body sizes, we classified nerve cells of the various nerve nuclei in the special sensory system into three groups. These data are of interest to better understand the process of nerve conduction in the special sensory system.
\end{abstract}

There have been several qualitative reports on the morphology of various brainstem nuclei in humans ${ }^{1,2)}$. Quantitative evaluations, however, are fairly rare, especially with methods minimizing shrinkage ratio. The aim of this study is to bring to light morphological and morphometrical comparisons of various nuclei in the special sensory system.

The special sensory system consists of the visual pathway (lateral geniculate body), the auditory pathway (ventral cochlear nucleus; dorsal cochlear nucleus; medial superior olivary nucleus; inferior collicular nucleus; medial geniculate body), the gustatory pathway (solitary tract nucleus) and the olfactory pathway. We studied morphological and morphometrical data regarding the abovementioned nuclei.

\section{Methods}

Using an image-analyzer equipped with a microscope and tissue sections stained with a special method (modified Klüver-Barrera staining method [K-B] after secondary fixation in chromic acid and embedding in celloidine ${ }^{3)}$, we examined the nerve cells in the special sensory system. We studied the brainstem nuclei after making serial K-B sections
(40 $\mu \mathrm{m}$ thick) of the brainstem and cerebellum in a 63-year-old female. Neurons for each nucleus (39 to 269 in number) were selected for measurement.

For the measurement of the neuronal areas, an image analyzing system was adopted: Soft Imaging System GmbH equipped with a microscope (Olympus BX 40) and a computer (Dell Precision 530). Enlarged images (40 times) of the neurons on the liquid crystalline viewer were measured with a pencil-type tracer (Wacom FAVO F630) to trace the contour of neurons having a nucleolus in the nucleus.

\section{Results}

Neuromorphological comparisons (see Fig. 1)

The lateral geniculate nucleus showed six laminations of neurons when it was observed on the coronary section: Layers 1-6 from the bottomup. Among these layers, layers 1 and 2 consisted of larger oval medium size neurons (LGN1 and LGN2), while layers 4 and 5 consisted of rather smaller oval medium size neurons (LGN4 and LGN5).

In the inferior collicular nucleus (ICN), the majority of neurons were small, oval, pyriform, spindle

Correspondence: Naoko Nonaka, D.D.S., Ph.D., Dept. of Anatomy, Showa University School of Medicine and Dept. of Oral Anatomy, Showa University School of Dentistry, 5-8, Hatanodai 1, Shinagawa-ku, Tokyo 142-8555, Japan. 


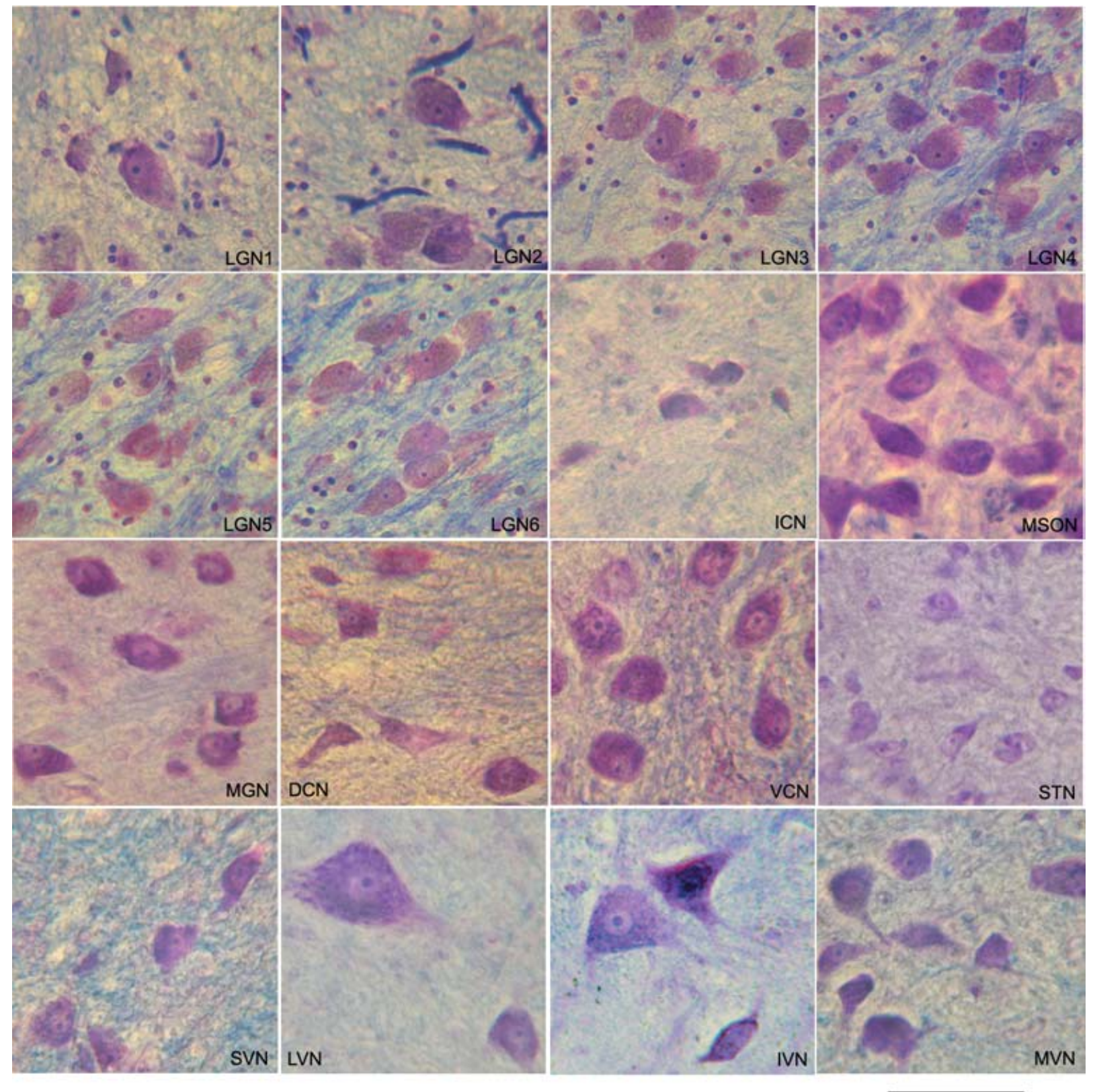

Fig. 1. Representative neurons of various nuclei in the human special sensory system. Modified K-B stain, scale bar $=50 \mu \mathrm{m}$.

Abbreviations: DCN: dorsal cochlear nucleus; ICN: inferior collicular nucleus; IVN: inferior vestibular nucleus; LGN1-6: Layer 1-6 of lateral geniculate nucleus; LVN: lateral vestibular nucleus; MGN: medial geniculate nucleus; MSON: medial superior olivary nucleus; MVN: medial vestibular nucleus; STN: solitary tract nucleus; SVN: superior vestibular nucleus; VCN: ventral cochlear nucleus.

shaped or triangular. In the center of the nucleus, there were medium sized, oval or triangular neurons.

The neurons in the medial superior olivary nucleus, many of them spindle shaped, medium sized neurons were gathered close to each other in a small area (MSON).

Neurons in the medial geniculate nucleus were oval and medium sized (MGN).
The dorsal cochlear nucleus differed in internal architecture from the ventral cochlear nucleus (DCN and VCN). The shape of neurons in DCN were medium sized, predominantly spindle shaped, while the neurons in VCN were characteristic, of medium size, plump, oval or round.

The solitary tract neurons were medium sized, oval and similar in shape (STN). 
Table 1. Morphometric comparison of nerve cells in the special sensory system

\begin{tabular}{lrr}
\hline Nucleus & $\mathrm{n}$ & $\begin{array}{c}\text { Cell body area }\left(\mu \mathrm{m}^{2}\right) \\
\text { mean } \pm \mathrm{SD}\end{array}$ \\
\hline Visual system-Lateral geniculate nucleus & \\
Layer 1 & 39 & $3,415.7 \pm 1,116.7$ \\
Layer 2 & 71 & $3,464.1 \pm 1,097.4$ \\
Layer 3 & 158 & $2,382.7 \pm 995.8$ \\
Layer 4 & 39 & $1,881.3 \pm 545.2$ \\
Layer 5 & 73 & $1,865.4 \pm 533.2$ \\
Layer 6 & 112 & $2,017.1 \pm 464.1$ \\
Auditory system & & \\
Inferior collicular nucleus & 90 & $1,204.7 \pm 732.3$ \\
Medial superior olivary nucleus & 139 & $2,372.2 \pm 776.0$ \\
Medial geniculate nucleus & 61 & $2,015.6 \pm 613.8$ \\
Dorsal cochlear nucleus & 110 & $2,020.9 \pm 524.4$ \\
Ventral cochlear nucleus & 167 & $3,256.8 \pm 1,007.0$ \\
Vestibular system & & \\
Superior vestibular nucleus & 62 & $2,540.3 \pm 658.5$ \\
Lateral vestibular nucleus & 63 & $4,605.0 \pm 2,470.9$ \\
Inferior vestibular nucleus & 71 & $5,356.0 \pm 1,778.9$ \\
Medial vestibular nucleus & 152 & $2,627.6 \pm 1,133.0$ \\
Gustatory system & & \\
Solitary tract nucleus & 269 & $2,618.8 \pm 1,304.6$ \\
\hline & & \\
\hline
\end{tabular}

The neurons in the superior vestibular nucleus were spindle shaped, oval, triangular or slender (SVN).

The lateral vestibular nucleus consists of two parts: the large neurons formed the lateral subnucleus; the medial subnucleus was composed of a large number of medium sized neurons (LVN).

The neurons were rather large sized, triangular, oval or multipolar, in inferior vestibular nucleus (IVN).

The majority of neurons were slender, oval, triangular or spindle shaped, in medial vestibular nucleus (MVN).

\section{Morphometrical comparisons}

After observation and measurements, the neurons in the special sensory system were classified into three groups: (1) Large size neurons (lateral vestibular nucleus: average 4,605.0 $\pm 2,470.9 \mu \mathrm{m}^{2}$ in area; inferior vestibular nucleus: average $5,356.0 \pm 1,778.9 \mu \mathrm{m}^{2}$ ); (2) medium size neurons, ranging in average areas from $3,464.1 \pm 1,097.4$ $\mu \mathrm{m}^{2}$ to $1,881.3 \pm 545.2 \mu \mathrm{m}^{2}$ (lateral geniculate nucleus, medial superior olivary nucleus, dorsal cochlear nucleus, ventral cochlear nucleus, superior vestibular nucleus, medial vestibular nucleus, solitary tract nucleus); and (3) small size neurons (inferior collicular nucleus: average 1,204 \pm 732.3 $\mu \mathrm{m}^{2}$ ). The measured data for each nucleus were listed in Table 1. There were differences in the mean cell body area among layers of lateral geniculate nucleus. The mean cell sizes were larger in layers 1 and 2 than those in the other layers.

\section{Discussion}

There are special peripheral apparatus for the reception of special sensations: eyes, ears, equilibrium apparatus, olfactory epithelium and taste buds. The signals from the above-mentioned apparatus are conducted to the brain by appropriate nerves. On the way to or inside the brain, nervous pathways play an important role in the relay of stimuli from peripheral ganglion cells to central nervous nuclei. We mainly studied the relay nuclei of the special sensory system in the brain, as listed in Table 2.

In the visual system, we studied the cytoarchitecture of lateral geniculate nucleus. The cell body areas of oval medium size neurons in layers 1 and 2 were larger than those in layers 4 and 5. This observation well corresponded to the difference between magnocellular and parvocellular layers. We also observed slight size differences between layers 3 and 4 , and between layers 5 and 6 . Layers 1, 4 and 6 receive nerve fibres from the contralateral eye, while layers 2,3 and 5 have connection with the homolateral retina. Further investigation is required to determin if such slight size differences relate to some visual functions.

Table 2. List of special sensory system

\begin{tabular}{llll}
\hline System & Special sense apparatus & Peripheral ganglia & Central nervous relay nuclei* \\
\hline Visual system & Rod cells \& cone cells & Retinal ganglion cells & LGN \\
Auditory system & Spiral organ (Corti) & Spiral ganglion (cochlea) & VCN, DCN, MSON, ICN, MGN \\
Vestibular system & $\begin{array}{l}\text { Maculae staticae } \\
\text { Crista ampularis }\end{array}$ & $\begin{array}{l}\text { Vestibular ganglion } \\
\text { (internal auditory meatus) }\end{array}$ & SVN, LVN, IVN, MVN \\
Gustatory system & $\begin{array}{l}\text { Taste buds } \\
\text { (caliculi gustatorii) }\end{array}$ & Geniculate ganglion & STN \\
Olfactory system & Olfactory cells & - & Olfactory bulb** \\
\hline
\end{tabular}

*Refer to the legend to Fig. 1 for abbreviations. **Not studied in this report. 
In the auditory system, the nerve fiber connection forms an auditory pathway: spiral ganglion $\rightarrow$ ventral or dorsal cochlear nucleus $\rightarrow$ (trapezoid body) $\rightarrow$ medial superior olivary nucleus $\rightarrow$ (lateral lemniscus) $\rightarrow$ inferior collicular nucleus $\rightarrow$ (inferior collicular brachium) $\rightarrow$ medial geniculate nucleus $\rightarrow$ (acoustic radiation) $\rightarrow$ transverse tegmental gyrus (Heschl). The neurons in the relay nuclei described above were of different sizes: for example, between the ventral and dorsal cochlear nuclei; between the ventral or dorsal cochlear nuclei and the medial superior olivary nucleus; between the medial superior olivary nucleus and the inferior collicular nucleus; between the inferior collicular nucleus and the medial geniculate nucleus.

In the vestibular system, there were notable differences in the cell body area among four nuclei. The cell body areas in lateral and inferior vestibular nuclei were extremely large, while those in superior and medial vestibular nuclei were smaller. The cell body area may relate to the conduction velocity of its axon, in the analogy of known response properties of parvocellular and magnocellular layers of the geniculate.
The data from this morphometric evaluation will no doubt be help in nerve conduction studies such as electric response audiometry. Although in the motor system $^{4,5)}$, a powerful muscle is basically supplied by a large neuron, it is not known if such theory can be applied or not to the special sensory system.

\section{References}

1) Olszewski J and Baxter D. Cytoarchitecture of the human brain stem. S. Karger, Basel-Munchen-Paris-London-New York-Sydney, 1954 \& 1982.

2) Goto N. Atlas of the human brainstem and cerebellum. Medical Tribune, Tokyo, 1989.

3) Goto N. Discriminative staining methods for the nervous system: Luxol fast blue-periodic acid Schiff-hematoxylin triple stain and subsidiary staining methods. Stain Technol 1987; 62:305-315.

4) Setoyama S, Zhou M, Goto N and Shimada K. Morphometric comparison of the motor trigeminal neurons. Okajimas Folia Anat Jpn 1997; 73:301-312.

5) Yuan H, Ke M, Goto N, Goto J and Suzuki K. Morphometric evaluation of the human cervical motoneurons. Okajimas Folia Anat Jpn 2000; 76:277-284. 